\title{
Supervivencia de implantes dentales en pacientes con infección por $\mathrm{VIH}$
}

\author{
Survival of dental implants in HIV-infected patients
}

Pamela Ponce Marón ${ }^{1 a}$

Edwin Ramos-Pilco ${ }^{1 a}$

Alejandro Aldana Cáceres ${ }^{2 a c}$

Gustavo Allasi Tejada ${ }^{1 a b}$

Correspondencia: edy4208@gmail.com
(7) https://orcid.org/0000-0002-5400-7895

(i) https://orcid.org/0000-0001-6750-8878

(D) https://orcid.org/0000-0001-7758-1411

(b) https://orcid.org/0000-0002-3371-7003

\section{Resumen}

Objetivo: Identificar la tasa de supervivencia de implantes dentales en pacientes con infección por el virus de inmunodeficiencia humana (VIH). Material y métodos: Se realizó una búsqueda electrónica de estudios observacionales y experimentales publicados hasta marzo del 2021 en la base de datos PubMed, PubMed Central, Cochrane y en las revistas de Periodoncia e Implantes con mayor factor de impacto según ISI Web of Science. La evaluación del riesgo de sesgo de los artículos observacionales se evaluó mediante la escala Newcastle-Ottawa y el manual Cochrane Versión 5.1.0 para ensayos clínicos. Resultados: Se encontraron 08 estudios observacionales, 02 reportes de caso, 05 series de casos y 01 estudio de cohorte con un seguimiento de 1 a 12 años. Se colocaron un total de 1033 implantes dentales, el conteo de linfocitos CD4+ presentó una media de $531.48 \mathrm{~mm}^{3}$. La tasa de supervivencia de implantes dentales en pacientes con VIH positivos fue de $95.31 \%$. Según la escala Newcastle-Ottawa, todos los artículos incluidos mostraron un bajo riesgo de sesgo. Conclusión: La presente revisión sistemática evidenció una tasa de supervivencia de los implantes dentales en pacientes con $\mathrm{VIH}$ positivos de $95.31 \%$ con un tiempo de seguimiento de 1 a 12 años.

Palabras clave: $\mathrm{VIH}$, terapia antiretroviral, implante dental, complicaciones

\section{Abstract}

Objective: To identify the survival rate of dental implants in patients with human immunodeficiency virus (HIV) infection. Material and methods: An electronic search was carried out for observational and experimental studies published up to march 2021 in the PubMed, PubMed Central, Cochrane databases and in the journals about periodontics and implants with a high impact factor according to ISI Web of Science. The assessment of the risk of bias of the observational articles was assessed using the Newcastle-Ottawa scale and the Cochrane manual Version 5.1.0 for clinical trials. Results: 8 observational studies, 2 case reports, 5 case series and 1 cohort study were found, with a follow-up of 1 to 12 years. A total of 1033 dental implants were placed, the CD4+ lymphocyte count presented a mean of $531.48 \mathrm{~mm} 3$. The dental implant survival rate in HIV positive patients was $95.31 \%$. According to the Newcastle Ottawa scale, all included articles showed a low risk of bias. Conclusion: The present systematic review showed a $95.31 \%$ dental implant survival rate in HIV positive patients with a follow-up time of 1 to 12 years.

Keywords: HIV, antiretroviral therapy, dental implant, complications

\footnotetext{
${ }^{1}$ Universidad Privada de Tacna. Facultad de Ciencias de la Salud. Programa de Segunda Especialidad en Periodoncia e Implantología. Tacna, Perú

2 Universidad Nacional Jorge Basadre Grohmann. Escuela Profesional de Odontología. Tacna, Perú

${ }^{a}$ Cirujano Dentista. Especialista en Periodoncia e implantes

${ }^{\mathrm{b}}$ Magíster en Salud Pública

${ }^{\mathrm{c}}$ Doctor en Ciencias de la Salud
} 


\section{Introducción}

El programa conjunto de las Naciones Unidas sobre el VIH/SIDA (ONUSIDA) estima que 38 millones de personas viven con el virus de la inmunodeficiencia humana $(\mathrm{VIH}) .^{1}$ En Perú, los casos notificados hasta el año 2020 fueron de 132657 infectados por VIH y 45089 casos de SIDA. ${ }^{2}$

La enfermedad se caracteriza por un fallo progresivo del sistema inmunológico que da lugar al desarrollo de infecciones oportunistas y neoplasias. El virus invade linfocitos T CD4+, macrófagos y células dendríticas, y el recuento de células T CD4+ disminuye gradualmente como resultado de la acción citopática directa o del ataque mediado por linfocitos T CD8+ citotóxico. La inmunidad celular se ve afectada una vez que el recuento de linfocitos ha descendido hasta situarse en un punto crítico y el paciente se vuelve vulnerable a infecciones oportunistas. ${ }^{3}$

Estudios han vinculado la presencia de VIH/SIDA a un mayor riesgo de complicaciones en procedimientos quirúrgicos orales ${ }^{4,5} \mathrm{El}$ riesgo elevado de complicaciones puede comprometer la supervivencia del implante dental y contribuir a los fracasos; sin embargo, como resultado de la introducción del tratamiento antirretroviral de gran actividad (TARGA), el VIH/SIDA se ha convertido en una enfermedad crónica y la esperanza de vida de los pacientes ha aumentado debido a un incremento en su resistencia inmunológica. ${ }^{4}$ Como resultado es probable que los pacientes busquen tratamiento dental, incluidos los implantes dentales para la rehabilitación oral y mejorar su calidad de vida.

El objetivo de la presente revisión sistemática fue identificar la tasa de supervivencia de los implantes dentales colocados en pacientes VIH positivos.

\section{Material y método}

\section{Protocolo y pregunta de investigación}

Se desarrolló un protocolo a priori con el objetivo de responder la siguiente pregunta ¿Cuál es la tasa de supervivencia de los implantes dentales colocados en pacientes con infección por VIH?

Este protocolo cumplió con las recomendaciones PRISMA (Preferred Reporting Items for Systematic Reviews and Meta-Analyses). ${ }^{6}$

\section{Extracción de datos}

\section{Criterios de inclusión "PICOS"}

- Población: Pacientes adultos VIH (+) con terapia antirretroviral.

- Intervención: Colocación de implantes dentales en pacientes VIH (+).

- Comparación: Colocación de implantes dentales en pacientes VIH (-).

- Resultados: Supervivencia del implante dental con un seguimiento desde el primer año.

- Diseño de estudio: Reportes de caso (5 o más casos), series de casos, estudios longitudinales prospectivos o retrospectivos, ensayos clínicos aleatorizados.

Criterios de inclusión: Revisiones sistemáticas; estudio en animales; estudios in vitro. 


\section{Tipos de resultados}

El objetivo primario de esta revisión sistemática fue evaluar la supervivencia de los implantes dentales en pacientes con $\mathrm{VIH}$.

El objetivo secundario fue evaluar cambios clínicos y/o complicaciones.

\section{Búsqueda electrónica y manual}

Se realizó una estrategia de búsqueda en las bases de datos PubMed Central, PubMed y biblioteca Chocrane hasta abril del 2021. Se utilizó los siguientes términos: (((("hiv") OR "human immunodeficency virus") OR "aids") OR "acquired immunodeficency syndrome")) AND "dental implants".

Además, se complementó con una búsqueda manual de las revistas de mayor impacto en periodoncia e implantología oral como Journal of Clinical Periodontology, Journal of Periodontology, Clinical Oral Implants Research, International Journal of Oral \& Maxillofacial Implants, European Journal of Oral Implantology, Implant Dentistry, International Journal of Periodontics and Restorative Dentistry, International Journal of Prosthodontics, Journal of Prosthetic Dentistry and Clinical Implant Dentistry and Related Research.

\section{Selección de artículos y extracción de datos}

Dos revisores de manera independiente (P.P. y E.R.) examinaron los títulos, resumen, resultados y conclusiones de artículos potenciales que fueron identificados. Sí hubo desacuerdo entre los revisores respecto a la selección de algún artículo; se realizó una discusión para llegar a un consenso. Cuando no se pudo alcanzar un consenso, un tercer revisor fue consultado (G.A.). La confiabilidad entre los revisores fue calibrada mediante el cálculo de porcentajes de acuerdo a los coeficientes Kappa (0.8 Kappa).

\section{Riesgo de sesgo}

Se evaluó el riesgo de sesgo de los artículos incluidos utilizando la escala de Newcastle-Ottawa (NOS), ${ }^{7}$ que evaluó tres categorías (selección, comparabilidad y resultados) y nueve asignaciones. Según la NOS se puede dar un máximo de nueve estrellas a un estudio, puntuaciones mayores o iguales a seis estrellas se consideró un bajo riesgo de sesgo, mientras que puntuaciones menores o iguales a cinco se valoró que el estudio presenta alto riesgo de sesgo.

\section{Resultados}

La búsqueda de la literatura, realizada de manera independiente por los revisores (P.P. y E.R.), logró un hallazgo de 850 artículos, de los que se seleccionaron 69, de los cuales 57 estudios fueron descartados después de la evaluación de títulos y resúmenes. A continuación, se examinó el texto completo de 12 artículos; por último, un total de 08 estudios cumplieron los criterios de inclusión y se analizaron en la presente revisión sistemática, de los cuales cinco artículos son series de casos, ${ }^{8-12}$ dos reportes de caso ${ }^{13,14}$ y un estudio de cohorte retrospectivo. ${ }^{15} \mathrm{El}$ diagrama de flujo se presenta en la Figura 1. 


\section{Figura 1}

Diagrama de flujos (formato PRISMA) filtración y proceso de selección ${ }^{14}$

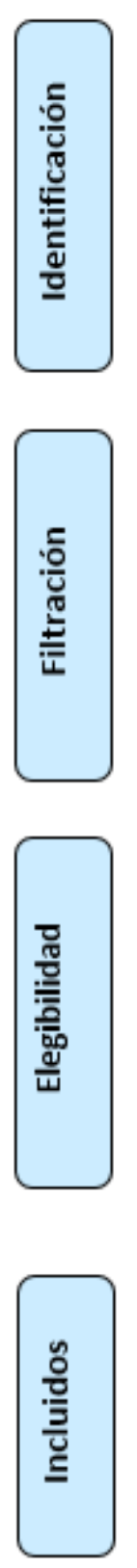

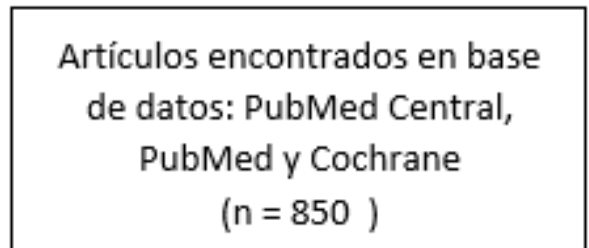

$(n=850)$
Artículos encontrados en otras fuentes científicas

$$
(n=04)
$$

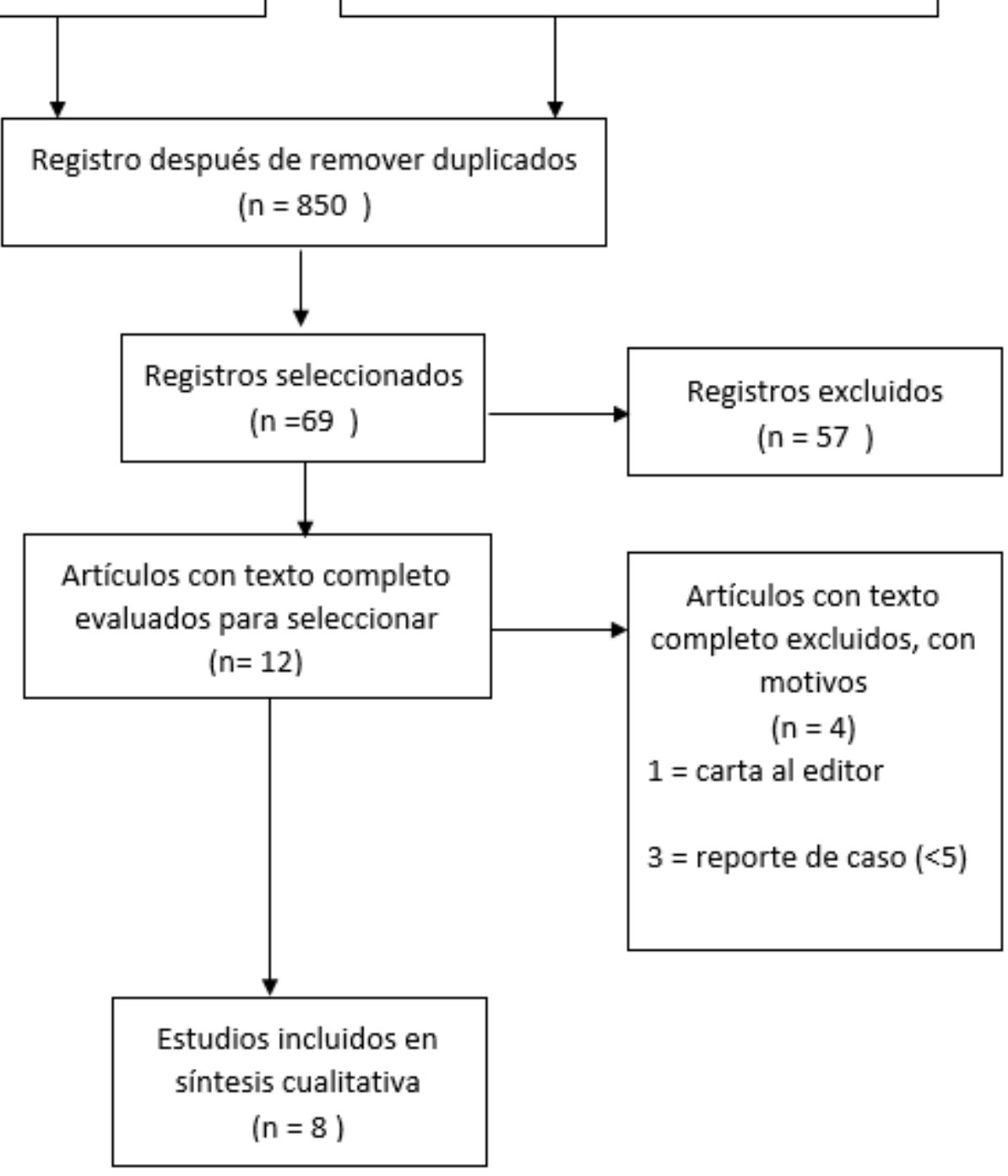

La evaluación del riesgo de sesgo de los estudios observacionales se muestra en la Tabla 1 y Figura 2, según la escala Newcastle-Ottawa (NOS) los ocho estudios incluidos presentaron un mínimo de seis estrellas, considerándose de bajo riesgo de sesgo. 
Tabla 1

Evaluación del riesgo de sesgo, escala Newcastle-Ottawa

\begin{tabular}{|c|c|c|c|c|c|c|c|c|c|c|c|}
\hline \multirow[b]{2}{*}{ Estudio } & \multicolumn{4}{|c|}{ Selección } & \multicolumn{3}{|c|}{ Comparabilidad } & \multicolumn{2}{|c|}{ Resultados } & \multirow[b]{2}{*}{ Total } & \multirow[b]{2}{*}{$\begin{array}{l}\text { Riesgo } \\
\text { de } \\
\text { sesgo }\end{array}$} \\
\hline & $\begin{array}{l}\text { Represen- } \\
\text { tatividad }\end{array}$ & $\begin{array}{l}\text { Grupo } \\
\text { control }\end{array}$ & $\begin{array}{l}\text { Determina- } \\
\text { ción } \\
\text { exposición }\end{array}$ & $\begin{array}{c}\text { Resultado } \\
\text { de interés } \\
\text { no estuvo } \\
\text { al inicio } \\
\end{array}$ & $\begin{array}{l}\text { Fact or } \\
\text { principal }\end{array}$ & $\begin{array}{l}\text { F a c t or } \\
\text { adicional }\end{array}$ & $\begin{array}{l}\text { Evaluación } \\
\text { resultado }\end{array}$ & $\begin{array}{l}\text { Seguimiento } \\
\mathrm{p} / \text { producir } \\
\text { resultados }\end{array}$ & $\begin{array}{c}\text { Adecuación } \\
\text { de } \\
\text { seguimiento }\end{array}$ & & \\
\hline $\begin{array}{l}\text { Oliveira } \\
\text { MA. } 2020\end{array}$ & $\stackrel{4}{4}$ & --- & 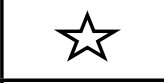 & 出 & --- & --- & 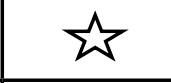 & 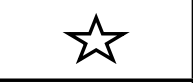 & $\stackrel{s}{3}$ & 6 & Bajo \\
\hline $\begin{array}{l}\text { Sabbah A. } \\
2019\end{array}$ & 光 & $\boldsymbol{W}$ & 象 & 象 & 象 & --- & 趈 & 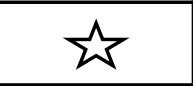 & $\stackrel{4}{3}$ & 8 & Bajo \\
\hline $\begin{array}{l}\text { Capparé P. } \\
2019\end{array}$ & $\hat{\psi}$ & --- & 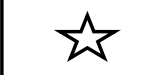 & 出 & --- & --- & 出 & 光 & 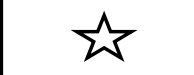 & 6 & Bajo \\
\hline $\begin{array}{l}\text { Rubinstein } \\
\text { NC. } 2019\end{array}$ & 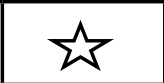 & -- & 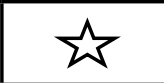 & 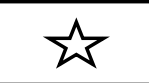 & --- & --- & 茨 & 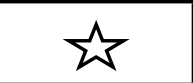 & $\stackrel{4}{3}$ & 6 & Bajo \\
\hline $\begin{array}{l}\text { Gastaldi } \\
\text { G. } 2017\end{array}$ & 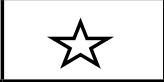 & -- & $\stackrel{4}{3}$ & 幽 & --- & --- & 幽 & $\sum$ & 象 & 6 & Bajo \\
\hline $\begin{array}{ll}\text { May } & \text { C. } \\
2016 & \\
\end{array}$ & 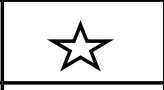 & -- & 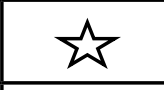 & 出 & --- & --- & 出 & 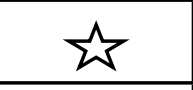 & 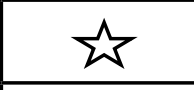 & 6 & Bajo \\
\hline $\begin{array}{l}\text { Gay-Esco- } \\
\text { da C. } 2016 \\
\end{array}$ & 光 & --- & 光 & 幽 & --- & --- & 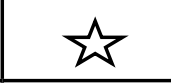 & 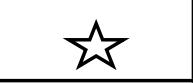 & 幽 & 6 & Bajo \\
\hline $\begin{array}{l}\text { Gherlone } \\
\text { E. } 2015\end{array}$ & 出 & --- & 光 & 象 & --- & -- & « & 出 & $\hat{\imath}$ & 6 & Bajo \\
\hline
\end{tabular}

\section{Figura 2}

A y $B$, evaluación del riesgo de sesgo según escala Newcastle-Ottawa

A

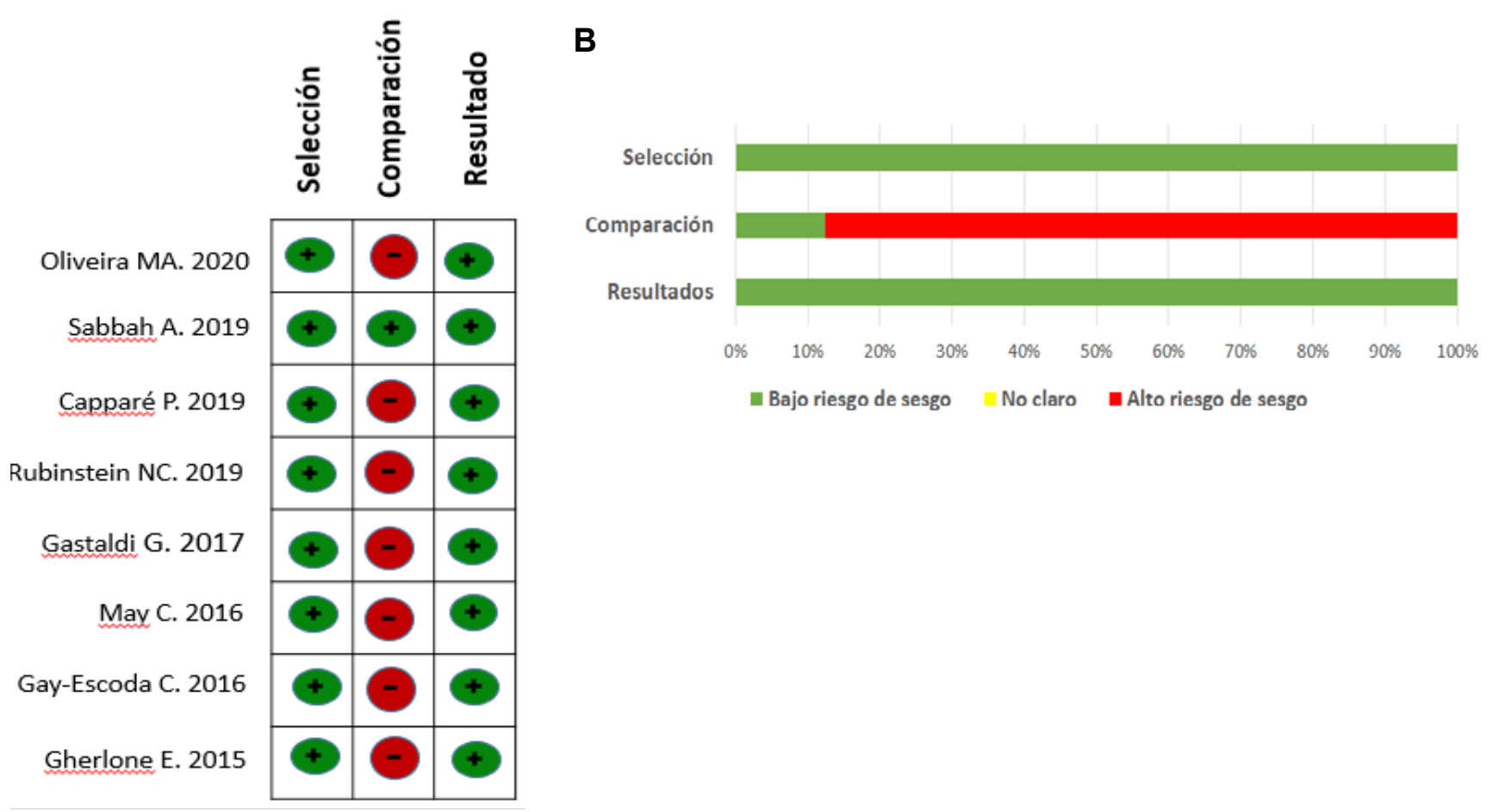


Se colocaron un total de 1033 implantes dentales en pacientes VIH positivos y 805 implantes dentales en pacientes $\mathrm{VIH}$ negativos. El conteo de linfocitos $\mathrm{CD}^{+}$(linfocitos $\mathrm{T} 4$ ) en sangre de sujetos VIH positivos tuvo una media de $531.48 \mathrm{~mm}^{3}$ con un rango de $141.25^{11}$ hasta $798.79 \mathrm{~mm}^{3}, 8$ la tasa de supervivencia de los implantes dentales colocados en pacientes $\mathrm{VIH}(+)$ varió desde $90.9 \%{ }^{11}$ hasta $100 \%,{ }^{9,13}$ mostrando una media de $95.31 \%$ con un tiempo de seguimiento de 1 a 12 años. La reabsorción ósea periimplantar fue en promedio de $0.93 \mathrm{~mm}$ a los dos años, ${ }^{10} 1.73 \mathrm{~mm}$ a los siete años ${ }^{8}$ y $2.43 \mathrm{~mm}$ a los doce años. ${ }^{13}$ Los estudios de Sabbah et al. (2019) y Gherlone et al. (2016) mencionaron que la tasa de fracaso de los implantes dentales colocados en pacientes $\mathrm{VIH}(+)$ fue por la condición de fumadores $(p<0.05)$, mientras que Sabbah et al. (2019) y May et al. (2016) mencionaron que el fracaso se evidenció en los implantes colocados en el maxilar anterior $(p<0.05)$.

Además, el estudio de Sabbah et al. (2019), que registró 484 implantes dentales colocados, mencionó un predictor de fracaso en sujetos $\mathrm{VIH}$ positivos que recibieron tratamiento de inhibidores de proteasa $(\mathrm{PI})$ con un $\mathrm{HR}=2.74, \mathrm{p}=0.04$. Para mayor información ver la Tabla 2.

Tabla 2

Formato PICO (P: población, I: intervención, C: comparación, O: resultados)

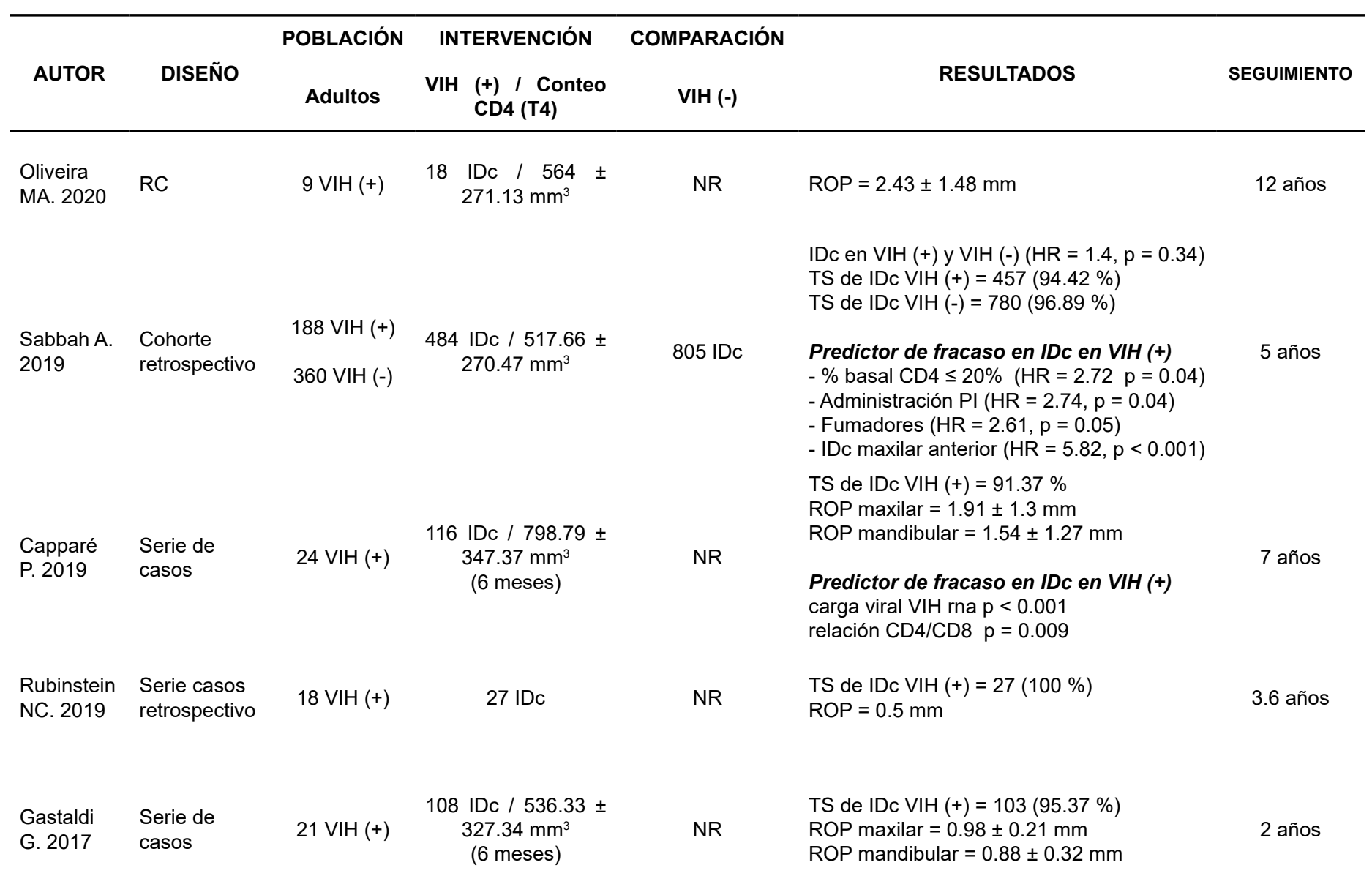




\begin{tabular}{|c|c|c|c|c|c|c|}
\hline $\begin{array}{l}\text { May C. } \\
2016\end{array}$ & $\begin{array}{l}\text { Serie de } \\
\text { casos }\end{array}$ & $16 \mathrm{VIH}(+)$ & $\begin{array}{c}33 \text { IDc / } 141.25 \pm \\
35.5 \mathrm{~mm}^{3}\end{array}$ & NR & $\begin{array}{l}\text { Predictor de fracaso en IDc en VIH (+) } 2 \\
\text { de } 3 \text { fracasos fueron maxilar anterior } \\
\text { TS de IDc VIH }(+)=30(90.9 \%)\end{array}$ & 5 años \\
\hline $\begin{array}{l}\text { Gay- } \\
\text { Escoda C. } \\
2016\end{array}$ & retrospectivo & $9 \mathrm{VIH}(+)$ & $57 \mathrm{IDc} / 436 \mathrm{~mm}^{3}$ & NR & TS de IDc = $56(98.3 \%)$ & $\begin{array}{r}\text { promedio } \\
\text { años }\end{array}$ \\
\hline $\begin{array}{l}\text { Gherlone } \\
\text { E. } 2015\end{array}$ & $\begin{array}{l}\text { Serie de } \\
\text { casos }\end{array}$ & $66 \mathrm{VIH}(+)$ & $\begin{array}{l}190 \text { IDc / } 726.3 \pm \\
201.4 \mathrm{~mm}^{3}\end{array}$ & NR & $\begin{array}{l}\text { Predictor de fracaso en IDc en VIH (+) } \\
\text { Fumadoresa } p \leq 0.005 \\
\text { TS de IDc } \mathrm{VIH}(+)=175(92.1 \%)\end{array}$ & 1 año \\
\hline
\end{tabular}

Nota. a: heavy smokers; RC: reporte de caso IDc: Implantes dentales colocados; ROP: reabsorción ósea periimplantar; NR: no reportado; TS: tiempo de supervivencia; PI: inhibidores de la proteasa; HR: Hazard ratio.

\section{Discusión}

\section{Calidad de la evidencia y sesgos potenciales en el proceso de revisión}

En la presente revisión sistemática, todos los artículos incluidos son estudios observacionales que corresponden a diseños de investigación, cuyo objetivo es la observación y registro de acontecimientos sin intervenir en estos; ${ }^{16}$ de acuerdo a la evaluación mediante la escala Newcastle-Ottawa, ${ }^{7}$ todos los artículos incluidos mostraron un bajo riesgo de sesgo (mayor o igual a seis estrellas), lo que pone en evidencia que las metodologías empleadas en estos estudios son reproducibles.

\section{Hallazgos comparados con otros estudios}

La tasa de supervivencia de los implantes dentales en pacientes VIH positivos de los artículos observacionales incluidos mostró una media de $95.31 \%$, resultados similares a los encontrados por Sivakumar et al. (2021), donde se reportó una tasa de superviviencia de $95 \%$. En comparación al grupo de pacientes VIH negativos estos resultados son similares a los encontrados por Howe et al. (2019) en una revisión sistemática donde se colocaron 2688 implantes dentales con una tasa de supervivencia media de $96.4 \%$.

La prueba de carga viral mide la cantidad de VIH que está presente en la sangre; dicha prueba se utiliza como marcador y verifica la progresión de la enfermedad. Se informó una asociación entre la carga viral y los resultados clínicos. ${ }^{17}$ Un aumento de la carga viral suele ir seguido de una disminución del recuento de CD4 ${ }^{+}$y enfermedad posterior, solo Capparé et al. (2019) reportaron el aumento de carga viral como predictor de fracaso de implantes dentales en pacientes VIH positivos $(p<0.001)$. El recuento de $C D 4^{+}$en el presente estudio registró una media de $531.48 \mathrm{~mm}^{3}$ (rango de 141.25 hasta $798.79 \mathrm{~mm}^{3}$ ).

A nivel de los sujetos que reciben el TARGA (tratamiento antirretroviral de gran actividad), solo Sabbah et al. (2019) informaron una tasa de fracaso en pacientes VIH positivos que recibieron los inhibidores de la proteasa (IP) $(H R=2.74, p=0.04)$, los demás estudios no reportaron diferencias significativas entre otros regímenes de TARGA. Se necesitarían más estudios para confirmar el resultado y dilucidar el mecanismo de acción. 
La reabsorción ósea periimplantar, en la presente revisión sistemática, fue de $0.93 \mathrm{~mm}$ a los 2 años, ${ }^{9}$ $1.73 \mathrm{~mm}$ en promedio a los 7 años ${ }^{8}$ y $2.43 \mathrm{~mm}$ a los 12 años. ${ }^{18}$ Estos resultados son similares a los encontrados en la literatura científica en pacientes VIH negativos que reportan una reabsorción ósea de 0.05-1.5 mm al primer año, seguido de una reabsorción de 0.05-0.2 mm/año..$^{19,20}$

Otra posible causa de fracaso en la oseointegración de los implantes dentales en pacientes VIH positivos fue el consumo de tabaco. Sabbah et al. (2019) y Gherlone et al. (2016) reportaron como predictores de fracaso a los pacientes fumadores $(H R=2.61 ; p<0.05)$. El monóxido de carbono presenta mayor afinidad por la hemoglobina, reduciendo la oxigenación de los tejidos cicatrizados; por su parte, la nicotina es vasoconstrictor e incrementa la agregación y adherencia plaquetaria, reduciendo el flujo sanguíneo y alterando la cicatrización de los tejidos, dando como resultado un mayor riesgo de mucositis, periimplantitis y pérdida del implante dental. ${ }^{21}$

En base a los resultados encontrados en la presente revisión, se propone las siguientes recomendaciones para el procedimiento de cirugía de implantes dentales:

- Consentimiento informado.

- Pacientes deben presentar salud periodontal.

- Pruebas de laboratorio: Linfocitos T-CD4+, carga viral (VIH), pruebas de coagulación sanguínea.

- Cirugía convencional para implantes dentales.

- Mínimo de torque de $30 \mathrm{~N} / \mathrm{cm}^{2}$.

- Enjuagatorio con digluconato de clorhexidina al $0.12 \%$, dos veces al día, por 10 días.

- Prescripción de antibióticos, solo en pacientes con alto riesgo de infección, debido a un estado inmunológico comprometido u otras afecciones sistémicas.

- Los implantes deben cargarse a los 4 meses.

- Mantenimiento periodontal, control radiográfico y pruebas serológicas cada seis meses.

\section{Limitaciones de los artículos incluidos}

La presente revisión sistemática presenta una evidencia científica limitada por el hecho de incluir estudios observacionales, estos estudios se pueden utilizar para informar resultados en los ámbitos del tratamiento y la prevención, la etiología, daño o morbilidad; el diagnóstico y el pronóstico e historia natural; escenarios en los que los estudios observacionales otorgan distintos niveles de evidencia, según el diseño en particular y el ámbito que se trate.

\section{Conclusiones}

La tasa de supervivencia de los implantes dentales en pacientes VIH positivos, en el presente estudio, mostró una media de $95.31 \%$ con un tiempo de seguimiento de 1 a 12 años.

Los probables factores predictores de fracasos de implantes dentales colocados fueron los pacientes fumadores y los implantes dentales colocados en el maxilar anterior. 


\section{Referencias}

1. United Nations AIDS Report. [Internet]. 2019 [citado 16 de abril de 2021]. Disponible en: https:// www.unaids.org/es/Homepage

2. Centro Nacional de Epidemiología, Prevención y Control de Enfermedades. Situación epidemiológica del VIH-Sida en el Perú. febrero de 2020;

3. Douek DC, Roederer M, Koup RA. Emerging concepts in the immunopathogenesis of AIDS. Annu Rev Med. 2009;60:471-84.

4. Patton LL, Shugars DA, Bonito AJ. A systematic review of complication risks for HIV-positive patients undergoing invasive dental procedures. J Am Dent Assoc 1939. febrero de 2002;133(2):195-203.

5. Campo J, Cano J, del Romero J, Hernando V, Rodríguez C, Bascones A. Oral complication risks after invasive and non-invasive dental procedures in HIV-positive patients. Oral Dis. enero de 2007;13(1):110-6.

6. Moher D, Liberati A, Tetzlaff J, Altman DG, PRISMA Group. Preferred reporting items for systematic reviews and meta-analyses: the PRISMA statement. PLoS Med. 2009;6(7):e1000097.

7. Ottawa Hospital Research Institute [Internet]. 2020 [citado 10 de mayo de 2021]. Disponible en: http://www.ohri.ca/programs/clinical_epidemiology/oxford.asp

8. Capparé P, Teté G, Romanos GE, Nagni M, Sannino G, Gherlone EF. The «All-on-four» protocol in HIV-positive patients: A prospective, longitudinal 7-year clinical study. Int J Oral Implantol Berl Ger. 2019;12(4):501-10.

9. Rubinstein NC, Jacobson Z, McCausland GL, Dibart S. Retrospective study of the success of dental implants placed in HIV-positive patients. Int J Implant Dent. 13 de junio de 2019;5(1):30.

10. Gastaldi G, Vinci R, Francia MC, Bova F, Capparé P. Immediate fixed rehabilitation supported by axial and tilted implants of edentulous jaws: a prospective longitudinal study in HIV-positive patients. J Osseointegration. 29 de junio de 2017;9(2):239-44.

11. May MC, Andrews PN, Daher S, Reebye UN. Prospective cohort study of dental implant success rate in patients with AIDS. Int J Implant Dent. diciembre de 2016;2(1):20.

12. Gherlone EF, Capparé P, Tecco S, Polizzi E, Pantaleo G, Gastaldi G, et al. A prospective longitudinal study on implant prosthetic rehabilitation in controlled HIV-Positive patients with 1-year follow-up: the role of CD4+ level, smoking habits, and oral hygiene. Clin implant dent relat res. octubre de 2016;18(5):955-64.

13. Oliveira MA de, Pallos D, Mecca F, Karem López Ortega null, Gallottini M, Andre Luiz Costa null, et al. Dental implants in patients seropositive for HIV: A 12-year follow-up study. J Am Dent Assoc 1939. noviembre de 2020;151(11):863-9.

14. Gay-Escoda C, Pérez-Álvarez D, Camps-Font O, Figueiredo R. Long-term outcomes of oral rehabilitation with dental implants in HIV-positive patients: A retrospective case series. Med Oral Patol Oral Cirugia Bucal. 1 de mayo de 2016;21(3):e385-391.

15. Sabbah A, Hicks J, MacNeill B, Arbona A, Aguilera A, Liu Q, et al. A retrospective analysis of dental implant survival in HIV patients. J Clin Periodontol. marzo de 2019;46(3):363-72.

16. Manterola C, Otzen T. Estudios observacionales: Los diseños utilizados con mayor frecuencia en investigación clínica. Int J Morphol. junio de 2014;32(2):634-45.

17. Shoko C, Chikobvu D. A superiority of viral load over CD4 cell count when predicting mortality in HIV patients on therapy. BMC Infect Dis. 15 de febrero de 2019;19(1):169.

18. Oliveira MA, Gallottini M, Pallos D, Maluf PSZ, Jablonka F, Ortega KL. The success of endosseous implants in human immunodeficiency virus-positive patients receiving antiretroviral therapy: a pilot study. J Am Dent Assoc 1939. septiembre de 2011;142(9):1010-6.

19. Albrektsson T, Zarb G, Worthington P, Eriksson AR. The long-term efficacy of currently used dental 
implants: a review and proposed criteria of success. Int J Oral Maxillofac Implants. 1986;1(1):11-25.

20. Hämmerle $\mathrm{CH}$, Brägger U, Bürgin W, Lang NP. The effect of subcrestal placement of the polished surface of ITI implants on marginal soft and hard tissues. Clin Oral Implants Res. junio de 1996;7(2):111-9.

21. Sgolastra F, Petrucci A, Severino M, Gatto R, Monaco A. Periodontitis, implant loss and peri-implantitis. A meta-analysis. Clin Oral Implants Res. abril de 2015;26(4):8-16

- Conflicto de intereses: La presente investigación no presenta conflicto de intereses entre los investigadores.

- Fuente de financiamiento: La presente investigación fue financiada por los investigadores. 